H. Umemura

Nagoya Math. J.

Vol. 77 (1980), 47-60

\title{
MODULI SPACES OF THE STABLE VECTOR BUNDLES OVER ABELIAN SURFACES
}

\author{
HIROSHI UMEMURA
}

Let $X$ be a projective non-singular variety and $H$ an ample line bundle on $X$. The moduli space of $H$-stable vector bundles exists by Maruyama [4]. If $X$ is a curve defined over $C$, the structure of the moduli space (or its compactification) $M(X, d, r)$ of stable vector bundles of degree $d$ and rank $r$ on $X$ is studied in detail. It is known that the variety $M(X, d, r)$ is irreducible. Let $L$ be a line bundle of degree $d$ and let $M(X, L, r)$ denote the closed subvariety of $M(X, d, r)$ consisting of all the stable bundles $E$ with $\operatorname{det} E=L$. We know the global Torelli theorem holds for the mapping $X \mapsto M(X, L, r)$ if the genus $g$ of $X \geq 2$ and $(d, r)=1$. Namely, let $X^{\prime}$ be a non-singular projective curve of genus $g$ and $L^{\prime}$ be a line bundle of degree $d$ on $X^{\prime}$. Then the variety $M(X, L, r)$ is isomorphic to $M\left(X^{\prime}, L^{\prime}, r\right)$ if and only if $X$ is isomorphic to $X^{\prime}$. In higher dimensional case, very little is known about the moduli space of $H$-stable vector bundles. The moduli spaces have been studied only on two types of surfaces. When $X$ is a hyperelliptic surface, we determined the moduli spaces of $H$-stable vector bundles with trivial Chern classes in Umemura [14]. In this case the Moduli spaces are not connected when we fixed the numerical Chern classes. Barth [1] proved the moduli space of $H$-stable vector bundles with $c_{1}=0$ of rank 2 over $P_{2}$ is irreducible and rational. In this paper, we work over abelian surfaces $A$ and we study the moduli spaces of some $H$-stable vector bundles. In the first example, a component of the moduli spaces is isomorphic to $A \times A$ and in the second example, it is birationally isomorphic to the symmetric product $S^{n}(A)$. In both cases, the local Torelli theorem holds (see for precise statements, Theorem 5 and Theorem 21). We know nothing about the connectedness of the moduli spaces.

Let $X$ be a non-singular algebraic surface defined over an algebraically closed field $k$ and $H$ an ample line bundle over $X$. We know that, if we

Received November 4, 1978. 
fix numerical Chern classes $c_{1}, c_{2}$ and a number $r$, the coarse moduli space $M\left(c_{1}, c_{2}\right)$ of $H$-stable vector bundles of rank $r$ exists. The moduli space $M\left(c_{1}, c_{2}\right)$ is a scheme of finite type over $k$ and the Zariski tangent space $T_{x}$ at a closed point $x \in M\left(c_{1}, c_{2}\right)$ is isomorphic to $H^{1}(X$, End $E)$ where $E$ is the $H$-stable vector bundle corresponding to the point $x$ (see Maruyama [4]). The scheme consisting of all the irreducible components of $M\left(c_{1}, c_{2}\right)$ passing through $x$ is called the moduli space of $E$ and denoted by $X(E)$. From now on, we assume all schemes are defined over $\boldsymbol{C}$.

LEMMA 1. Let $Y$ be an irreducible non-singular algebraic variety and $\mathscr{E}$ be a family of $H$-stable vector bundles over $X$ parametrized by $Y$, i.e. $\mathscr{E}$ is a locally free sheaf over $Y \times X$ and for any closed point $y \in Y$, the vector bundle $E_{y}$ over $y \times X$ is $H$-stable. Let $f: Y \rightarrow M\left(c_{1}, c_{2}\right)$ denote the map defined by $\mathscr{E}$. We assume that, for any closed point $y \in Y$, $\operatorname{dim} H^{1}\left(X\right.$, End $\left.E_{y}\right)=$ $\operatorname{dim} Y$. If $f$ is injective, then $f$ is an open immersion. In particular $Y$ is birationally equivalent to an irreducible component of $M\left(c_{1}, c_{2}\right)$.

Proof. In fact, for any closed point $y \in Y, \operatorname{dim} H^{1}\left(X\right.$, End $\left.E_{y}\right)=\operatorname{dim} Y$ $\leq \operatorname{dim} X \leq \operatorname{dim} H^{1}\left(X\right.$, End $\left.E_{y}\right)$. Hence $\operatorname{dim} f(Y)=\operatorname{dim} X$ and $f(X)$ is contained in the open subset of nonsingular points of $M\left(c_{1}, c_{2}\right)$. Therefore the analytic map $f^{a n}: X^{a n} \rightarrow M\left(c_{1}, c_{2}\right)^{a n}$ is an open immersion. It follows from Mumford [9], $f$ is étale. Now the lemma follows.

Lemma 2. Let $A$ be an abelian surface (abelian variety of dimension 2), $H$ an ample line bundle over $A$ and $E$ an $H$-stable vector bundle over $A$. If $E$ is absolutely simple, of type $M_{0}$ and a model, then there is an injection $\check{A} \times A \rightarrow X(E)$.

Proof. Let $\mathscr{L}$ be the Poincaré line bundle over $A \times \check{A}, p: A \times(\check{A} \times A)$ $\rightarrow A$ be the map defined by

$$
p((a, b, c))=a+c,
$$

and $p_{12}: A \times(A \times A) \rightarrow A \times A$ be the projection. Consider the vector bundle $\mathscr{E}=p^{*} E \otimes p_{12}^{*} \mathscr{L}$ over $A \times(\check{A} \times A)$. This is a family of $H$-stable vector bundles over $A$ parametrized by $\check{A} \times A$. The vector bundle over a point $(L, a) \in \check{A} \times A$ is $T_{a}^{*} E \otimes L$. Let $f: \check{A} \times A \rightarrow X(E)$ be the map defined by $\mathscr{E} . \quad$ If $T_{a}^{*} E \otimes L \simeq T_{a^{\prime}}^{*} E \otimes L^{\prime}, \quad T_{a}^{*} E \simeq T_{a^{\prime}}^{*} E \otimes L^{\prime} \otimes L^{-1}$ hence $T_{a-a^{\prime}}^{*} E$ $\simeq E \otimes L^{\prime} \otimes L^{-1}$. In particular $T_{a-a^{\prime}}^{*} P(E) \simeq P(E)$. The hypothesis that $E$ is a model implies $H(P(E))=0$ where $H(P(E))=\{x \in A \mid P(E) \simeq$ 
$\left.T_{x}^{*} P(E)\right\}$ (Umemura [15]). Hence $a=a^{\prime}$. We get $E \otimes L \simeq E \otimes L^{\prime}$. It. follows from the absolute simplicity of $E$ that $L \simeq L^{\prime}$. This proves $f$ is injective. See Umemura [15].

Let $F$ be an $V$-stable vector bundle with numerical Chern classes $c_{1}$, $c_{2}$ and of rank $r$ over an abelian surface $A$. Let $y \in M\left(c_{1}, c_{2}\right)$ be the corresponding point. Let us put $\Delta(F)=(r-1) c_{1}^{2}-2 r c_{2} . \quad-\Delta(F)$ is equal to the second Chern class of End $F$.

LEMMA 3. The dimension of the Zariski tangent space at $y$ is equal to $-\Delta(F)+2$.

Proof. It follows from the Riemann-Roch theorem, $\operatorname{dim} H^{\circ}(A$, End $F)$. $-\operatorname{dim} H^{1}(A$, End $F)+\operatorname{dim} H^{2}(A$, End $F)=-c_{2}($ End $F)=\Delta(F)$. By the Serre duality $\operatorname{dim} H^{0}(A$, End $F)=\operatorname{dim} H^{2}(A$, End $F)$. Since a stable bundle is simple, $\operatorname{dim} H^{0}(A$, End $F)=1$. Hence $\operatorname{dim} H^{1}(A$, End $F)=-\Delta(F)+2$ which is equal to the dimension of the Zariski tangent space at $y$.

LEMMA 4. Under the same hypothesis as in Lemma 2, moreover if $\Delta(E)=-2$, then $X(E)$ is irreducible and $\check{A} \times A$ is isomorphic to $X(E)$.

Proof. Let $x^{\prime} \in X(E)$ and $E^{\prime}$ the corresponding $H$-stable bundle. By Lemma $3 \operatorname{dim} H^{1}\left(A\right.$, End $\left.E^{\prime}\right)=-\Delta\left(E^{\prime}\right)+2$. Since $E^{\prime}$ has the same numerical Chern classes as $E,-\Delta\left(E^{\prime}\right)+2=-\Delta(E)+2=4$. Now it follows. from Lemmas 1 and 2 that the map $f$ constructed in the proof of Lemma 2 is an isomorphism.

ExAmple 1. Let $C$ be a non-singular projective curve of genus $2, P$ a point of $C$ and $J$ the Jacobian variety of $C$. Let $C^{(n)}$ be the $n$-th symmetric product of $C$. We assume $n \geq 3$. There is a projection $\varphi: C^{(n)} \rightarrow J$ defined by

$$
\varphi\left(Q_{1}+\cdots+Q_{n}\right)=\left(Q_{1}+\cdots+Q_{n}-n P\right),
$$

where $Q_{i} \in C$ for $1 \leq i \leq n$. We know that there exists a vector bundle $E_{n-1}$ of rank $n-1$ over $J$ such that $C^{(n)}$ is $J$-isomorphic to $P\left(E_{n-1}\right)$. We proved in Umemura [4] that $E$ is $\mathcal{O}(C)$-stable, of type $M_{0}$ and a model. The number $\Delta(E)$ is also calculated -2 . Hence we can apply Lemma 4. The moduli space $X\left(E_{n-1}\right)$ is isomorphic to $\check{J} \times J$. The determinant defines. a map $\check{J} \times J \rightarrow \breve{J}\left(y \mapsto \operatorname{det} E_{y}\right)$. This map is surjective and all the fibers which we denote $X^{\prime}\left(E_{n-1}\right)$ are isomorphic to $J$. Hence we proved:

Theorem 5. The scheme $X^{\prime}\left(E_{n-1}\right)$ is isomorphic to J. The local Torelli theorem holds for $C \mapsto X^{\prime}\left(E_{n-1}\right)$. 
ExAmple 2. Let $A$ be an abelian surface and $L$ be a principal polarization of $A$, i.e., $L$ is ample and $\operatorname{dim} H^{\circ}(A, L)=1$. Let $n \geq 3$ be an integer and $L_{i}$ be a line bundle algebraically equivalent to $L$ and $\varphi_{i}$ a non-zero section of $L_{i}, 1 \leq i \leq n . \varphi_{i}$ is uniquely determined up to the multiplication of a non-zero constant. Let $E\left(L_{1}, L_{2}, \cdots, L_{n}\right)$ be the coherent sheaf over $A$ defined by the exact sequence:

$$
\begin{aligned}
0 \rightarrow \mathcal{O} & \rightarrow L_{1} \oplus L_{2} \oplus \cdots \oplus L_{n} \rightarrow E\left(L_{1}, L_{2}, \cdots, L_{n}\right) \rightarrow 0, \\
1 & \mapsto\left(\varphi_{1}, \varphi_{2}, \cdots, \varphi_{n}\right) .
\end{aligned}
$$

The coherent sheaf $E\left(L_{1}, L_{2}, \cdots, L_{n}\right)$ does not depend on the choice of $\varphi_{i}$. For, let $\varphi_{i}^{\prime}$ be another non-zero section of $L_{i}$. There exists a non-zero constant $c_{i}$ such that $\varphi_{i}^{\prime}=c_{i} \varphi_{i}$. Hence the diagram

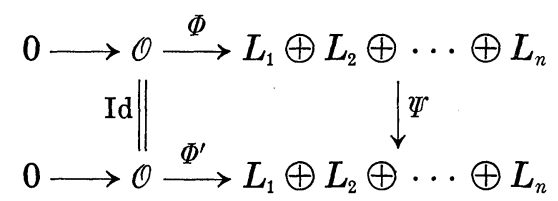

is commutative where $\Phi$ is the injection of the exact sequence (i), $\Phi^{\prime}$ is the injection obtained from $\Phi$ by replacing $\varphi_{i}$ by $\varphi_{i}^{\prime}$ and $\Psi$ is the $\mathcal{O}_{A}$-linear map defined by the diagonal matrix

$$
\left[\begin{array}{llll}
c_{1} & & & \\
& c_{2} & & \\
& & \ddots & \\
& & & c_{n}
\end{array}\right] .
$$

Let $C$ be an irreducible non-singular projective curve of genus 2 . Let $P$ be a point of $C$ and $\varphi$ the map of $C$ to $J$ defined by $\varphi(Q)=(Q-P)$. We denote by $C$ the image $\varphi(C)$. Let $P_{1}, P_{2}, \cdots, P_{6}$ be the points of $C$ such that $\mathcal{O}\left(2 P_{i}\right)$ is isomorphic to the canonical bundle $K$ of $C$. Let $C_{i}$ be the image of $C$ in $J$ defined by $\varphi_{i}\left(Q-P_{i}\right)=\mathcal{O}\left(Q-P_{i}\right) \in J$ for $Q \in C$.

Lemma 5. If $x$ is a point of $J$ such that $x \notin \bigcup_{i=1}^{6} C_{i}$, then $C \cap(C+$ $x) \cap(C+2 x)=\varnothing$.

Proof. Suppose that $C \cap C+x \cap C+2 x$ is not empty. Then there exist three points $Q, Q^{\prime}, Q^{\prime \prime}$ of $C$ such that $\mathcal{O}(Q-P)=\mathcal{O}\left(Q^{\prime}-P\right)+x$, $\mathcal{O}(Q-P)=\mathcal{O}\left(Q^{\prime \prime}-P\right)+2 x$. Hence $\mathcal{O}\left(Q-Q^{\prime}\right)=x, \mathcal{O}\left(Q-Q^{\prime \prime}\right)=2 x$. Therefore $\mathcal{O}\left(2 Q-2 Q^{\prime}\right)=\mathcal{O}\left(Q-Q^{\prime \prime}\right)$. Finally we get $\mathcal{O}\left(Q+Q^{\prime \prime}-2 Q^{\prime}\right)=\mathcal{O}$. We 
study two cases separately.

Case (i) $K \neq \mathcal{O}\left(2 Q^{\prime}\right)$. In this case, by the Riemann-Roch theorem $\operatorname{dim} H^{0}\left(C, \mathcal{O}\left(2 Q^{\prime}\right)\right)=1$. Hence it follows from $\mathcal{O}\left(Q+Q^{\prime \prime}-2 Q^{\prime}\right)=\mathcal{O}$ that $Q+Q^{\prime \prime}=2 Q^{\prime}$ as divisors. Consequently $Q=Q^{\prime}$ and $Q^{\prime \prime}=Q^{\prime}$. This shows $x=0$. Hence $x \notin C_{i}$ for every $i$. This is a contradiction.

Case (ii) $K=\mathcal{O}\left(2 Q^{\prime}\right)$. Hence there exists an $i$ such that $Q^{\prime}=P_{i}$. Therefore $x=\mathcal{O}\left(Q-Q^{\prime}\right)=\mathcal{O}\left(Q-P_{i}\right)$ is in $C_{i}$. This is impossible.

Let $D$ be the effective divisor on $A$ such that $L \simeq \mathcal{O}(D)$. Such divisors are limited: (a) There exist a non-singular curve $C$ of genus 2 and a point $P$ of $C$ such that the abelian variety $A$ is isomorphic to the Jacobian variety $J$ of $C$ and $D$ coincides with $C$. (b) There exist two elliptic curves $C_{1}, C_{2}$ such that the abelian variety $A$ is isomorphic to $C_{1} \times C_{2}$ and $D$ is $C_{1} \times 0 \cup 0 \times C_{2}$ (Weil [18]). Let us study first the case (a).

Corollary 6. For any integer $N_{1}$, there exist an integer $N \geq N_{1}$ and a point $x \in J$ of order $N$ such that $C \cap(C+x) \cap(C+2 x)=\varnothing$.

This is an easy consequence of Lemma 5.

Lemma 7. Let $x$ be a point of the Jacobian variety $J(=A)$ of order $N \geq 3$ and $L \simeq \mathcal{O}(C)$. If $C \cap(C+x) \cap \cdots \cap(C+(N-1) x)=\varnothing$, then $E(L$, $\left.T_{x}^{*} L, \cdots, T_{(N-1) x}^{*} L\right)$ is an L-stable locally free sheaf.

Proof. The locally freeness of $E\left(L, T_{x}^{*} L, \cdots, T_{(N-1) x}^{*}\right)$ is evident. The cyclic group $(x)$ operates on the exact sequence;

(ii) $\quad 0 \rightarrow \mathcal{O} \rightarrow L \oplus T_{x}^{*} L \oplus \cdots \oplus T_{(N-1) x}^{*} L \rightarrow E\left(L, T_{x}^{*} L, \cdots, T_{(N-1) x}^{*} L\right) \rightarrow 0$.

Hence there exists an exact sequence of vector bundles over $A^{\prime}=A /(x)$;

$$
0 \rightarrow \mathcal{O} \rightarrow F \rightarrow E^{\prime} \rightarrow 0
$$

such that $\pi^{*}$ (iii) is isomorphic to (ii) where $\pi$ is the isogeny $A \rightarrow A /(x)$. $F$ is nothing but the direct image $\pi_{*} L$ (Morikawa [7]). Hence by Takemoto [11], $F$ is $\operatorname{det} F$-stable. The line bundle $\operatorname{det} F$ will be $\operatorname{denoted}$ by $L^{\prime}$. Suppose that $E^{\prime}$ is not $L^{\prime}$--stable. Then there exist a non-zero locally free sheaf $G^{\prime}$ of rank $<N-1$ and a morphism $g^{\prime}$ of $\mathcal{O}_{A^{\prime}}$-modules $E^{\prime} \rightarrow G^{\prime}$ such that $g^{\prime}$ is surjective on $A^{\prime}-($ a subvariety of codimension $\geq 2$ ) and such that

$$
\frac{\left(c_{1}\left(E^{\prime}\right) \cdot L^{\prime}\right)}{r\left(E^{\prime}\right)} \geq \frac{\left(c_{1}\left(G^{\prime}\right) \cdot L^{\prime}\right)}{r\left(G^{\prime}\right)} .
$$


Since $F$ is $L^{\prime}$-stable,

$$
\frac{\left(c_{1}\left(F^{\prime}\right) \cdot L^{\prime}\right)}{r\left(F^{\prime}\right)}<\frac{\left(c_{1}\left(G^{\prime}\right) \cdot L^{\prime}\right)}{r\left(G^{\prime}\right)} .
$$

Let now $M^{\prime}$ be a line bundle over $A^{\prime}$. Then $N\left(M^{\prime} \cdot L^{\prime}\right)=\pi^{*}\left(M^{\prime} \cdot L^{\prime}\right)=$ $\left(\pi^{*} M^{\prime} \cdot L^{\otimes N}\right)=N\left(\pi^{*} M^{\prime} \cdot L\right)$. Hence we proved $\left(\pi^{*} M^{\prime} \cdot L\right)=\left(M^{\prime} \cdot L^{\prime}\right)$. Applying this rule to the inequalities (iv) and (v), we get

$$
\frac{\left(L^{\otimes N} \cdot L\right)}{N}<\frac{\left(c_{1}(G) \cdot L\right)}{r(G)} \leq \frac{\left(L^{\otimes N} \cdot L\right)}{N-1}
$$

where $G=\pi^{*} G^{\prime}$. Consequently

$$
2<\frac{\left(c_{1}(G) \cdot L\right)}{r(G)} \leq 2+\frac{2}{N-1} .
$$

Since $r(G)<N-1$, it follows that $\left(c_{1}(G) \cdot L\right)=2 r(G)+1$. If we put $\tilde{G}=$ $G \otimes L^{-1}$, then $\left(c_{1}(\tilde{G}) \cdot L\right)=1$. Let $\varphi$ be the isogeny $\mathrm{y} \mapsto N y$ of $A$ onto $A$ itself. Then, since the morphism induced by $g^{\prime} \varphi^{*}\left(L \oplus T_{x}^{*} L \oplus \cdots \oplus T_{(N-1) x}^{*} L\right)$ $\otimes L^{-1}=\overbrace{\mathscr{O} \oplus \cdots \oplus \mathcal{O}}^{N} \rightarrow \varphi^{*} G$ is surjective on $A-$ (a subvariety of codimension $\geqslant 2), H^{0}\left(A, \varphi^{*} G \operatorname{det} \tilde{G}\right) \neq 0$. On the other hand, since the spectral sequence degenerates, we get $H^{0}\left(A, \varphi^{*} \operatorname{det} \tilde{G}\right) \simeq H^{0}\left(A, \varphi_{*} \varphi^{*} \operatorname{det} \tilde{G}\right)=\bigoplus_{\varphi^{*} \mathscr{L}=0_{A}}$ $H^{\circ}(A, \mathscr{L} \otimes \operatorname{det} \tilde{G})$. Therefore, there exists a line bundle $\mathscr{L}$ on $A$ such that $\varphi^{*} \mathscr{L} \simeq \mathcal{O}_{A}, H^{0}(A, \mathscr{L} \otimes \operatorname{det} \tilde{G}) \neq 0$. Let $D$ be an effective divisor on $A$ such that $\varphi^{*} \mathscr{L}=\mathcal{O}_{A}, \mathcal{O}(D)=\mathscr{L} \otimes \operatorname{det} \tilde{G}$. Then $(D \cdot L)=1$. This is impossible as we proved in Umemura [16].

LEMMA 8. Using the same notation as in the preceding lemma, we assume moreover $N \geq 4$ and $C \cap(C+x) \cap(C+2 x)=\varnothing$. Then $E(L, T * L, \cdots$, $\left.T_{m x}^{*} L\right)$ is an L-stable locally free sheaf for $3 \leq m \leq N$.

Proof. We put $E_{r}=E\left(L, T_{x}^{*} L, \cdots, T_{(r+1) x}^{*} L\right)$ for $2 \leq r \leq N-1$. The local freeness of $E_{r}$ follows from the hypothesis $C \cap(C+x) \cap(C+2 x)=\varnothing$. Now we prove the $L$-stability of $E$ by the descending induction on $r$. Lemma 7 shows that $E_{N-1}$ is $L$-stable. Let us assume $E_{r}$ is $L$-stable for an $r, 3 \leq r \leq N-1$ and show $E_{r-1}$ is $L$-stable. The diagram

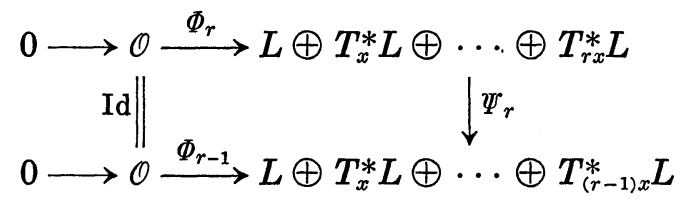


is commutative where $\Phi_{r}(1)=\left(\varphi, T_{x}^{*} \varphi, \cdots, T_{r x}^{*} \varphi\right), \quad 0 \neq \varphi \in H^{0}(A, L), \Phi_{r-1}(1)$ $=\left(\varphi, T_{x}^{*} \varphi, \cdots, T_{(r-1) x}^{*} \varphi\right)$ and $\Psi_{r}$ is the projection onto the first $r$ factors. The projection $\Psi_{r}$ induces a surjection: $\psi_{r}: E_{r} \rightarrow E_{r-1}$ and the Ker $\psi_{r}=$ $T_{r x}^{*} L$. Hence we get an exact sequence

$$
0 \rightarrow L^{\prime} \rightarrow E_{r} \rightarrow E_{r-1} \rightarrow 0
$$

where $L^{\prime}=T_{r x}^{*} L$. Tensoring $L^{\prime-1}$ with the exact sequence, we obtain a new exact sequence

$$
0 \rightarrow \mathcal{O} \rightarrow E_{r}^{\prime} \rightarrow E_{r-1}^{\prime} \rightarrow 0 \text {. }
$$

Our induction hypothesis is that $E_{r}^{\prime}$ is $L$-stable and we have to show $E_{r-1}^{\prime}$ is $L$-stable. Let $G$ be a non-zero locally free sheaf of rank $\leq r-1$ and $E_{r-1}^{\prime} \rightarrow G$ be a morphism which is surjective on $X$ - (a subvariety of codimension $\geq 2)$. By the stability of $E_{r}^{\prime},\left(c_{1}\left(E_{r}^{\prime}\right) \cdot L\right) / r\left(E_{r}^{\prime}\right)<\left(c_{1}(F) \cdot L\right) / r(G)$. Since $c_{1}\left(E_{r}^{\prime}\right)$ is algebraically equivalent to $L$, we get $2 / r<\left(c_{1}(F) \cdot L\right) / r(G)$. If $\left(c_{1}(F) \cdot L\right) \geq 2$, then

$$
\frac{\left(c_{1}\left(E_{r-1}^{\prime}\right) \cdot L\right)}{r\left(E_{r-1}^{\prime}\right)}=\frac{2}{r-1}<\frac{2}{r(G)} \leq \frac{\left(c_{1}(F) \cdot L\right)}{r(G)} .
$$

Hence we may assume $\left(c_{1}(F) \cdot L\right)=1$. Then there is a generically surjective homomorphism $\left(L \oplus T_{x}^{*} L \oplus \ldots \oplus T_{r x}^{*} L\right) \otimes T_{r x}^{*} L^{-1} \rightarrow G \otimes T_{r x}^{*} L^{-1}$. The argument of the proof of Lemma 7 shows this is impossible.

Let us now examine the case (b).

Lemma 9. Let $C_{1}, C_{2}$ be elliptic curves. $A=C_{1} \times C_{2}$ and $L=\mathcal{O}\left(C_{1} \times\right.$ $\left.0+0 \times C_{2}\right)$. Let $r$ be an integer $\geq 2$ and $x_{i}$ be a point of order $r+1$ of $C_{i}, 1 \leq i \leq 2$. If we put $x=\left(x_{1}, x_{2}\right)$, then $E\left(L, T_{x}^{*} L, \cdots, T_{r x}^{*} L\right)$ is an $L$ stable locally free sheaf.

Proof. Let us put $E=E\left(L, T_{x}^{*} L, \cdots, T_{r x}^{*} L\right), \quad C_{1} \times 0 \cup 0 \times C_{2}=D$. Since $D \cap T_{x}^{*} D \cap T_{2 x}^{*} D=\varnothing, E$ is locally free. Let us show the restriction $\left.E\right|_{C_{1} \times 0}$ (resp. $\left.E\right|_{0 \times C_{2}}$ ) on $C_{1} \times 0$ (resp. $0 \times C_{2}$ ) of $E$ is stable. We need

Sublemma 10. Let $C$ be an elliptic curve and $M$ a line bundle of degree 1 on $C$. Let $s$ be an integer $\geq 2$ and $y$ be a point of $C$ of order $s+1$. Let $E$ be the coherent sheaf defined by the following exact sequence;

$$
\begin{gathered}
0 \rightarrow \mathcal{O} \rightarrow L \oplus T_{y}^{*} L \oplus \cdots \oplus T_{s y}^{*} L \rightarrow E \rightarrow 0 \\
1 \mapsto\left(\psi, T_{y}^{*}, \cdots, T_{s y}^{*} \psi\right)
\end{gathered}
$$


where $0 \neq \psi \in H^{0}(C, L)$. Then $E$ is locally free and stable.

Proof of the sublemma. For the same reason as in the proof of the lemma, $E$ is locally free. As in the proof of Lemma 7 the cyclic group (y) operates on the exact sequence and there exists a exact sequence of vector bundles

$$
0 \rightarrow \mathcal{O} \rightarrow F \rightarrow E^{\prime} \rightarrow 0
$$

over $C^{\prime}=C /(y)$ such that $\pi^{*}$ (vii) is isomorphic to (vi) where $\pi$ is the isogeny $C \rightarrow C /(y)$. For the same reason as before, $F$ is stable. Let $E^{\prime} \rightarrow$ $G^{\prime}$ be a non-trivial quotient vector bundle. Since $F$ is stable, $1 /(s+1)$ $=d(F) / r(F)<d\left(G^{\prime}\right) / r\left(G^{\prime}\right)$. Hence $d\left(G^{\prime}\right) \geq 1$. Since $r\left(E^{\prime}\right)>r\left(G^{\prime}\right), d\left(E^{\prime}\right) / r\left(E^{\prime}\right)$ $=1 / r\left(E^{\prime}\right)<d\left(G^{\prime}\right) / r\left(G^{\prime}\right)$. This shows $E^{\prime}$ is stable. Since the degree of $\pi$ is $s$, and $s$ is relatively prime to $r(E)=s-1, \pi^{*} E^{\prime}=E$ is stable.

Let us come back to the proof of Lemma 9 . Let $G$ be a non-zero locally free sheaf of rank $r$ on $A$ and $E\left(L, T_{x}^{*} L, \cdots, T_{r x}^{*} L\right) \rightarrow G$ be a morphism which is surjective on $A-$ (a subvariety of codimension $\geq 2$ ). Since the restrictions are stable,

$$
\frac{\left(c_{1}(E) \cdot C_{1} \times 0\right)}{r(E)}=\frac{d\left(E \mid C_{1} \times 0\right)}{r(E)}<\frac{d\left(G \mid C_{1} \times 0\right)}{r(G)}=\frac{\left(c_{1}(G) \cdot C_{1} \times 0\right)}{r(G)}
$$

and

$$
\frac{\left(c_{1}(E) \cdot 0 \times C_{2}\right)}{r(E)}<\frac{\left(c_{1}(G) \cdot 0 \times C_{2}\right)}{r(G)} .
$$

Therefore

$$
\begin{aligned}
\frac{\left(c_{1}(E) \cdot L\right)}{r(E)}= & \frac{\left(c_{1}(E) \cdot C_{1} \times 0\right)}{r(E)}+\frac{\left(c_{1}(E) \cdot 0 \times C_{2}\right)}{r(E)}<\frac{\left(c_{1}(G) \cdot C_{1} \times 0\right)}{r(G)} \\
& +\frac{\left(c_{1}(G) \cdot 0 \times C_{2}\right)}{r(G)}=\frac{\left(c_{1}(G) \cdot L\right)}{r(G)} .
\end{aligned}
$$

Lemma 11. Let us assume that $E\left(L_{1}, L_{2}, \cdots, L_{n}\right)=E$ is locally free. Then the number $\Delta(E)=(r(E)-1) c_{1}(E)^{2}-2 r(E) c_{2}(E)$ is equal to $-2 n$.

Proof. The first Chern class $c_{1}(E)$ is numerically equivalent to $L^{\otimes n}$ and the second Chern class $c_{2}(E)$ is numerically equivalent to $(n(n-1) / 2) L^{2}$. Hence,

$$
\begin{aligned}
\Delta(E) & =(n-2) n^{2} L^{2}-2(n-1) \frac{n(n-1)}{2} L^{2} \\
& =2(n-2) n^{2}-2(n-1)^{2} n=-2 n .
\end{aligned}
$$


Remark 12. We do not know whether all $E\left(L_{1}, L_{2}, \cdots, L_{n}\right)=E$ is $L$ stable. We can prove the following assertion which will be used in the sequel.

Lemma 13. Suppose that if $i=j, L_{i} \neq L_{j}$. If $E\left(L_{1}, L_{2}, \cdots, L_{n}\right)=E$ is locally free, $E$ is simple $\left(\operatorname{dim} H^{0}(A\right.$, End $\left.E)=1\right)$.

Sublemma 14. For $1 \leq i \leq n, H^{0}\left(A, \check{E} \times L_{i}\right)=0$.

Proof. We have an exact sequence;

$$
0 \rightarrow \check{E} \rightarrow \check{L}_{1} \oplus \check{L}_{2} \oplus \ldots \oplus \check{L}_{n} \rightarrow \mathcal{O} \rightarrow 0 .
$$

Tensoring with $L_{i}$, we get

$$
0 \rightarrow \check{E} \oplus L_{i} \rightarrow\left(\check{L}_{1} \oplus \ldots \check{L}_{n}\right) \otimes L_{i} \rightarrow L_{i} \rightarrow 0 .
$$

The long exact sequence of cohomology group is;

$$
0 \rightarrow H^{0}\left(\check{E} \otimes L_{i}\right) \rightarrow H^{0}\left(\left(\check{L}_{1} \oplus \check{L_{2}} \oplus \cdots \oplus \check{L_{n}}\right) \otimes L_{i}\right) \rightarrow H^{0}\left(L_{i}\right) \rightarrow \cdots .
$$

From the hypothesis $H^{0}\left(\left(\check{L}_{1} \oplus \check{L}_{2} \oplus \cdots \oplus \check{L}_{n}\right) \otimes L_{i}\right) \simeq H^{0}(\mathcal{O})$ and the homomorphism $H^{0}\left(\left(\check{L}_{1} \oplus \check{L}_{2} \oplus \cdots \oplus \check{L}_{n}\right) \otimes L_{i}\right) \simeq H^{0}(\mathcal{O}) \rightarrow H^{0}\left(L_{i}\right)$ is not zero. Hence $H^{0}\left(A, \check{E} \otimes L_{i}\right)=0$.

Sublemma 15. $H^{l}\left(A, E \otimes L_{i}\right) \simeq H^{l}(\mathcal{O})$, for $l=1,2$.

Let us write the long exact sequence of cohomology of (ix) again;

$$
\begin{aligned}
0 & \rightarrow H^{0}\left(\left(\check{L}_{1} \oplus \check{L}_{2} \oplus \ldots \oplus \check{L}_{n}\right) \otimes L_{i}\right) \rightarrow H^{0}\left(L_{i}\right) \rightarrow H^{1}\left(\check{E} \otimes L_{i}\right) \\
& \rightarrow H^{1}\left(\left(\check{L}_{1} \oplus \check{L}_{2} \oplus \ldots \oplus \check{L}_{n}\right) \otimes L_{i}\right) \rightarrow H^{1}\left(L_{i}\right) \rightarrow H^{2}\left(\check{E} \otimes L_{i}\right) \\
& \rightarrow H^{2}\left(\left(\check{L}_{1} \oplus \check{L}_{2} \oplus \ldots \oplus \check{L}_{n}\right) \otimes L_{i}\right) \rightarrow H^{2}\left(L_{i}\right) .
\end{aligned}
$$

Now the assertion follows from the following;

(1) $H^{j}\left(\left(\check{L}_{1} \oplus \check{L}_{2} \oplus \cdots \oplus \check{L}_{n}\right) \otimes L_{i}\right)=H^{j}(\mathcal{O})$ for any $j$.

(2) $H^{1}\left(L_{i}\right)=0$ for $1=1,2$.

Sublemma 16. $H^{0}(A, \check{E})=0, H^{1}(A, \check{E}) \simeq H^{0}(\mathcal{O}), \operatorname{dim} H^{2}(A, \check{E})=n+1$.

The first two assertions follow easily from the long exact sequence of cohomology groups of the exact sequence (viii). The last assertion follows from the Riemann-Roch theorem for $\check{E}$.

Proof of Lemma 13. Tensoring $E$ with the exact sequence (viii), we get $(\mathrm{x})$

$$
0 \rightarrow \check{E} \otimes E \rightarrow \check{L}_{1} \otimes E \oplus \check{L}_{2} \otimes E \oplus \cdots \oplus \check{L}_{n} \otimes E \rightarrow E \rightarrow 0
$$


The last terms of the exact sequence are;

$$
\begin{aligned}
\cdots & \rightarrow H^{1}(E) \rightarrow H^{2}(\check{E} \otimes E) \\
& \rightarrow H^{2}\left(\check{L}_{1} \otimes E \oplus \check{L_{2}} \otimes E \oplus \cdots \oplus \check{L}_{n} \otimes E\right) \rightarrow H^{2}(E) .
\end{aligned}
$$

By the Serre duality and Sublemmas 15 and 16,

$$
\begin{aligned}
& \operatorname{dim} H^{1}(E)=\operatorname{dim} H^{1}(\check{E})=1, \\
& \operatorname{dim} H^{2}\left(L_{1} \otimes \check{E} \oplus L_{2} \otimes \check{E} \oplus \cdots \oplus L_{n} \otimes \check{E}\right) \\
& \quad=\operatorname{dim} H^{0}\left(\check{L}_{1} \otimes E \oplus \check{L}_{2} \otimes E \oplus \cdots \oplus \check{L}_{n} \otimes E\right)=0 .
\end{aligned}
$$

Hence $\operatorname{dim} H^{2}(\check{E} \otimes E) \leq 1$. By the Serre duality $\operatorname{dim} H^{\circ}(\check{E} \otimes E) \leq 1$. But $H^{\circ}(\check{E} \otimes E)$ contains $k$ as homothesies. Hence $\operatorname{dim} H^{\circ}(A, \check{E} \otimes E)=1$.

LEMma 17. If $E\left(L_{1}, L_{2}, \cdots, L_{n}\right) \simeq E\left(L_{1}^{\prime}, L_{2}^{\prime}, \cdots, L_{n}^{\prime}\right)$, the set $\left\{L_{1}, L_{2}, \cdots\right.$, $\left.L_{n}\right\}$ coincide with the set $\left\{L_{1}^{\prime}, L_{2}^{\prime}, \cdots, L_{n}^{\prime}\right\}$ counted with multiplicity.

In fact let $M$ be a line bundle algebraically equivalent to $L$. Tensoring $M^{-1}$. whth the exact sequence, we get

$$
\begin{aligned}
0 \rightarrow & M^{-1} \rightarrow\left(L_{1} \oplus L_{2} \oplus \cdots \oplus L_{n}\right) \otimes M^{-1} \\
& \rightarrow E\left(L_{1}, L_{2}, \cdots, L_{n}\right) \otimes M^{-1} \rightarrow 0 .
\end{aligned}
$$

Since $M$ is ample, $H^{0}\left(A, M^{-1}\right)=H^{1}\left(A, M^{-1}\right)=0$. Hence $H^{0}\left(\left(L_{1} \oplus L_{2} \oplus \ldots\right.\right.$ $\left.\left.\oplus L_{n}\right) \otimes M^{-1}\right) \simeq H^{0}\left(E\left(L_{1}, L_{2}, \cdots, L_{n}\right) \otimes M^{-1}\right)$. Since the dimension of $H^{0}\left(\left(L_{1}\right.\right.$ $\left.\oplus L_{2} \oplus \cdots \oplus L_{n}\right) \otimes M^{-1}$ ) is the number of times that $M$ appears is the set $L_{1}, L_{2}, \cdots, L_{n}$, the lemma follows.

Lemma 18. Let $M, M^{\prime}$ be line bundles algebraically equivalent to 0 . If $E\left(L_{1}, L_{2}, \cdots, L_{n}\right) \otimes M \simeq E\left(L_{1}^{\prime}, L_{2}^{\prime}, \cdots, L_{n}^{\prime}\right) \otimes M^{\prime}$, then $M \simeq M^{\prime}$ and $E\left(L_{1}\right.$, $\left.L_{2}, \cdots, L_{n}\right) \simeq E\left(L_{1}^{\prime}, L_{2}^{\prime}, \cdots, L_{n}^{\prime}\right)$.

Tensoring $M^{-1}$, we may assume $M^{\prime} \simeq \mathcal{O}$. Suppose that $M$ is not isomorphic to $\mathcal{O}$. Then tensoring $M$ with the exact sequence (i), we get

$$
0 \rightarrow M \rightarrow\left(L_{1} \oplus L_{2} \oplus \cdots \oplus L_{n}\right) \otimes M \rightarrow E\left(L_{1}, L_{2}, \cdots, L_{n}\right) \otimes M \rightarrow 0 .
$$

Since $M$ is algebraically equivalent to $0, H^{i}(A, M)=0$ for any $i$ and

$$
H^{0}\left(\left(L_{1} \oplus L_{2} \oplus \cdots \oplus L_{n}\right) \otimes M\right) \simeq H^{0}\left(E\left(L_{1}, L_{2}, \cdots, L_{n}\right) \otimes M\right) .
$$

Hence $\operatorname{dim} H^{0}\left(E\left(L_{1}, L_{2}, \cdots, L_{n}\right) \otimes M\right)=n$. On the other hand, from the exact sequence (i),

$$
\begin{aligned}
0 \rightarrow & H^{0}(\mathcal{O}) \rightarrow H^{0}\left(L_{1}^{\prime} \oplus L_{2}^{\prime} \oplus \cdots \oplus L_{n}^{\prime}\right) \rightarrow H^{0}\left(E\left(L_{1}^{\prime}, L_{2}^{\prime}, \cdots, L_{n}^{\prime}\right)\right) \\
& \rightarrow H^{1}(\mathcal{O}) \rightarrow H^{1}\left(L_{1}^{\prime} \oplus L_{2}^{\prime} \oplus \cdots \oplus L_{n}^{\prime}\right)=0 .
\end{aligned}
$$


Hence $\operatorname{dim} H^{0}\left(E\left(L_{1}^{\prime}, L_{2}^{\prime}, \cdots, L_{n}^{\prime}\right)\right)=n+1$ and $E\left(L_{1}, L_{2}, \cdots, L_{n}\right) \otimes M$ is not isomorphic to $E\left(L_{1}^{\prime}, L_{2}^{\prime}, \cdots, L_{n}^{\prime}\right)$.

Let now $p_{i n+1}: \overbrace{A \times \cdots \times A}^{n+1} \rightarrow A \times A(1 \leq i \leq n)$ be the projection onto the product of $i$-th and $(n+1)$-th factors. Let $m: A \times A \rightarrow A$ be the group law of $A$. Let $\mathscr{L}_{i}$ be the inverse image $\left(m \circ p_{i n+1}\right) * L$ and $\Theta_{i}=\left(m \circ p_{i n+1}\right) *(\theta)$ where $\theta$ is a fixed non-zero section of $L$. The coherent sheaf $\mathscr{E}$ on $\overbrace{A \times \cdots \times A}^{n+1}$ is defined by the exact sequence;

$$
\begin{gathered}
0 \rightarrow \mathcal{O} \rightarrow \mathscr{L}_{1} \oplus \mathscr{L}_{2} \oplus \cdots \oplus \mathscr{L}_{n} \rightarrow \mathscr{E} \rightarrow 0 \\
1 \mapsto\left(\Theta_{1}, \Theta_{2}, \cdots, \Theta_{n}\right)
\end{gathered}
$$

The coherent sheaf $\mathscr{E}$ is considered as a family of coherent sheaves on the last $A$ parametrized by the first $\overbrace{A \times \cdots \times A}^{n}$. Let $\left(x_{1}, x_{2}, \cdots, x_{n}\right)$ be a point of $\overbrace{A \times \cdots \times A}^{n}$. The restriction of the exact sequence (xii) to the fibre $\left(x_{1}, \cdots, x_{n}\right) \times A$ is

$$
\begin{aligned}
0 \rightarrow \mathcal{O} & \rightarrow T_{x_{1}}^{*} L+T_{x_{2}}^{*} L+\cdots+T_{x_{n}}^{*} L \rightarrow E\left(T_{x_{1}}^{*} L, T_{x_{2}}^{*} L, \cdots, T_{x_{n}}^{*} L\right) \rightarrow 0 \\
& 1 \mapsto\left(T_{x_{1}}^{*} \Theta_{1}, T_{x_{2}}^{*} \Theta_{2}, \cdots, T_{x_{n}}^{*} \Theta_{n}\right)
\end{aligned}
$$

The symmetric group $\varsigma_{n}$ operates on $\overbrace{A \times \cdots \times A}^{n}$ hence on $\overbrace{A \times \cdots \times A}^{n})$ $\times A$. There is an operation of $\widetilde{S}_{n}$ on $\mathscr{L}_{1} \oplus \mathscr{L}_{2} \oplus \cdots \oplus \mathscr{L}_{n}$ covering its operation on $(\overbrace{A \times \cdots \times A}^{n} A) \times A$. This operation is compatible with the injection $0 \rightarrow \mathscr{L}_{1} \oplus \mathscr{L}_{2} \oplus \cdots \oplus \mathscr{L}_{n}$. Hence $\widetilde{S}_{n}$ acts on $\mathscr{E}$. It follows from the descent theory that there exists a coherent sheaf $\mathscr{E}$ over $S^{n}(A) \times A$ such that $\pi^{*} \mathscr{E}^{\prime} \simeq \mathscr{E}$ where $S^{n}(A)$ is the $n$-th symmetric product of $A$ and $\pi: \overbrace{A \times \cdots \times A}^{n} \rightarrow S^{n}(A)$ is the projection.

Let $\mathscr{P}$ be the Poincaré line bundle over $\check{A} \times A \simeq A \times A$ and $p_{23}: S^{n}(A)$ $\times A \times A \rightarrow A \times A$ the projection. We put $p_{23}^{*} \mathscr{P}=\mathscr{P}^{\prime}$ and $\mathscr{E}^{\prime} \otimes \mathscr{P}^{\prime}=\mathscr{E}^{\prime \prime}$. Then $\mathscr{E}^{\prime \prime}$ is a family of coherent sheaves on $A$ parametrized by $S^{n}(A) \times A$. Let $(x, y),\left(x^{\prime}, y^{\prime}\right) \in S^{n}(A) \times A$. If $\left.\mathscr{E}^{\prime \prime}\right|_{(x, y) \times A}$ is isomorphic to $\left.\mathscr{E}^{\prime \prime}\right|_{(x, y) \times A}$, then $(x, y)=\left(x^{\prime}, y^{\prime}\right)$ by Lemma 17 and Lemma 18 .

Proposition 19. There exists a non-empty open subset $U$ of $S^{n}(A)$ such that (i) $\mathscr{E}^{\prime \prime} \mid U \times A \times A=\mathscr{E}^{(3)}$ is locally free, (ii) for any point $(x, y) \in U \times A$, $\mathscr{E}^{\prime \prime} \mid(x, y) \times A$ is L-stable. 
Proof. Let $X=\left\{(x, y, z) \in S^{n}(A) \times A \times A \mid \Theta_{i}(x, z)=0\right.$ for any $1 \leq i \leq n$. Then $X$ is a closed subset of $S^{n}(A) \times A \times A$. The coherent sheaf $\mathscr{E}^{\prime \prime}$ is locally free outside $X$. Let $p_{1}: S^{n}(A) \times A \times A \rightarrow S^{n}(A)$ be the projection onto the first factor. Since $p_{1}$ is proper, $p_{1}(X)$ is a proper closed subset of $S^{n}(A)$ by Corollary 6. Let $U^{\prime}=S^{n}(A)-p_{1}(X)$. Then $\mathscr{E}^{\prime \prime} \mid U^{\prime} \times A \times A$ is locally free. By Corollary 6, Lemma 8 and Lemma 9, there exists a point $x \in U^{\prime}$ such that $\mathscr{E}^{(3)} \mid(x, y) \times A$ is $L$-stable for any point $y \times A$. Since the stability is an open condition by Maruyama [4], there exists non-empty open subset $U$ of $U^{\prime}$ satisfying the condition (i) and (ii) of the proposition.

THEOREM 20. The algebraic variety $S^{n}(A) \times A$ is birationally isomorphic to a component of the moduli space of L-stable vector bundles.

Proof. Since $U \times A$ parametrizes a family of $L$-stable bundles, we get a morphism from $U \times A$ to a component of the moduli space. This map is injective by Lemma 17 and Lemma 18. The theorem now follows from Lemma 1, Lemma 3 and Lemma 11.

This component will be denoted by $X_{n}(A ; L)$. Let $g: X_{n}(A ; L) \rightarrow \check{A}=$ $A$ be the map such that $g(x)=(\operatorname{det} E) \otimes L^{-1}$. Since this map is birationally equivalent to $S^{n}(A) \times A \rightarrow A,\left(\left(x_{1}, \cdots, x_{r}\right), y\right) \mapsto \sum x_{j}+(n-1) y$ for $x \in \check{A}$ $=A$, the fibre $g^{-1}(z)=Y_{n}(A ; L)$ is birational to $Z^{n}(A)=\left\{\left(\left(x_{1}, \cdots, x_{n}\right), y\right)\right.$ $\left.\in S^{n}(A) \times A \mid \sum x_{i}+(n-1) y=0\right\}$.

Theorem 21. The map $(A, L) \mapsto Y_{n}(A, L)$ separates locally the moduli space of the principally polarized abelian surfaces.

Proof. Let $A, B$ be abelian varieties of any dimension. We put

$$
\begin{aligned}
& Z^{n}(A)=\left\{\left(\left(x_{1}, \cdots, x_{n}\right), y\right) \in S^{n}(A) \times A \mid \sum x_{i}+(n-1) y=0\right\}, \\
& Z^{n}(B)=\left\{\left(\left(x_{1}, \cdots, x_{n}\right), y\right) \in S^{n}(B) \times B \mid \sum x_{i}+(n-1) y=0\right\} .
\end{aligned}
$$

It is sufficient to show that, if $Z^{n}(A)$ is birational to $Z^{n}(B)$, then $A$ is isomorphic to $B$. This will be proved in Proposition 24 .

Let $X$ be an algebraic variety and $n>0$ an integer. The symmetric group $\mathfrak{S}_{n}$ acts on the product $\overbrace{X \times \cdots \times X}^{n}$. The quotient variety $\overbrace{X \times \cdots \times X}^{n} / \mathscr{S}_{n}$ is the $n$-th symmetric product of $X$ and denoted by $S^{n}(X)$.

Lemma 22. Let $A$ be an abelian variety. The closed subvariety $\left\{\left(x_{1}\right.\right.$, $\left.\left.x_{2}, \cdots, x_{n}\right) \in A \times \cdots \times A \mid \sum_{i=1}^{n} x_{i}=0\right\}$ of $S^{n}(A)$ will be denoted by $T^{n}(A)$. 
Let $\tilde{T}^{n}(A)$ be a non-singular model of $T^{n}(A)$. If $n \geq 2, H^{0}\left(\tilde{T}^{n}(A), \Omega^{1}\right)=0$.

Let $V$ be the universal covering space of $A$. Hence there exists a lattice $\Gamma \subset V$ such that $V / \Gamma \simeq A . \quad H^{1}(A, \mathcal{O}) \simeq T$ where $T=\operatorname{Hom}_{C}(V, C)$ (see Mumford [10]). By the Künneth formula, $H^{1}(A \times \cdots \times A, \mathcal{O}) \simeq T \oplus$ $\cdots \oplus T$. The symmetric group acts on $H^{1}(A \times \cdots \times A, \mathcal{O}) \simeq T \oplus \cdots \oplus T$ as permutation of factors. Let $U^{n}(A)$ denote the closed subvariety $\left\{\left(x_{1}, x_{2}\right.\right.$, $\left.\left.\cdots, x_{n}\right) \in A \times \cdots \times A \mid \sum_{i=1}^{n} x_{i}=0\right\}$ of $\overbrace{A \times \cdots \times A}^{n}$ and $i$ the inclusion $U^{n}(A) \smile \overbrace{A \times \cdots \times A}^{n} . \quad U^{n}(A)$ is isomorphic to $\overbrace{A \times \cdots \times A}^{n-1}$. Notice that the symmetric group $\mathfrak{S}_{n}$ operates on $U^{n}(A)$ and $U^{n}(A) / \widetilde{S}_{n}=T^{n}(A)$. It is easy to check that the restriction of the map $i^{*}: H^{1}(A \times \cdots \times A, \mathcal{O})$ $=\overbrace{T \oplus \cdots \oplus T}^{n} \rightarrow H^{1}\left(U^{n}(A), \mathcal{O}\right)=\overbrace{T \oplus \cdots \oplus T}^{n-1}$ to the subspace $U=\left\{\left(t_{1}, t_{2}\right.\right.$, $\left.\left.\cdots, t_{n}\right) \in T+\cdots+T \mid \sum_{i=1}^{n} t_{i}=0\right\}$ is an isomorphism. Hence $H^{1}\left(U^{n}(A), \mathcal{O}\right)^{\varsigma_{n}}$ $=U^{\Im_{n}}=0$. On the other hand $H^{1}\left(U^{n}(A), \mathcal{O}\right)^{\varsigma_{n}}=H^{1}\left(\tilde{T}^{n}(A), \mathcal{O}\right)$ by Proposition 9. 24, Ueno [12] hence the lemma is proved.

Lemma 23. Any rational map from $\tilde{T}^{n}(A)$ to $B$ is trivial, i.e., $f\left(\tilde{T}_{n}\right)$ ) is a point.

Proof. Blowing up the given $\tilde{T}^{n}(A)$ if necessary, we may assume $f$ regular. Suppose that the dimension of $f\left(T^{n}(A)\right)$ is positive. Then there exist a holomorphic 1-form $\omega$ on $B$ such that $f^{*} \omega \neq 0$ which conducts Lemma 22.

Proposition 24. Let $A, B$ be abelian varieties. If $Z^{n}(A)$ is birationally isomorphic to $Z^{n}(B)$, then $A$ is isomorphic to $B$.

Proof. If $n=1$, then $Z^{1}(A)=A, Z^{1}(B)=B$ and the assertion is well known (see. Weil [17]). We assume $n \geqq 2$. Let $f: Z^{n}(A) \rightarrow Z^{n}(B)$ be a birational map. Let $\pi_{A}$ (resp. $\pi_{B}$ ) be the map from $Z^{n}(A)$ to $A$ (resp. from $Z^{n}(B)$ to $\left.B\right)$ defined by $\pi_{A}\left(\left(x_{1}, \cdots, x_{n}\right), y\right)=\sum_{i=1}^{n} x_{i}$ (resp. $\pi_{B}\left(\left(x_{1}, \cdots, x_{n}\right), y\right.$ ) $\left.=\sum_{i=1}^{n} x_{i}\right)$. Then by Lemma 23, $f$ induces a birational map $\bar{f}: A \rightarrow B$ such that $\pi_{B} \circ f=\bar{f} \circ \pi_{A}$. Hence $A$ is biregularly isomorphic to $B$.

In our examples, the moduli spaces have irregularity 4. The abelian variety $A \times \hat{A}$ operates on the moduli spaces; $E \mapsto T_{X}^{*} E \otimes L, x \in A, L \in \hat{A}$. In most cases this operation is effective modulo finite group and hence by a theorem of Matsumura-Nishi [6], the irregularity of the moduli space $\geq 4$. 
Question 25. When is the irregularity of the moduli space 4?

\section{REFERENCES}

[1] Barth, W., Moduli of vector bundles on the projective plans, Inventiones Math., 42 (1977), 63-91.

[2] Maruyama, M., Stable vector bundles on an algebraic surface, Nagoya Math. J., 58 (1975), 25-68.

[ 3 ] - Openness of a family of torsion free sheaves, J. Math. Kyoto Univ., 16 (1976), 627-637.

[4] - Moduli of stable sheaves, I, II, J. Math. Kyoto Univ., 17 (1977), 91-126, preprint.

[5] - Boundedness of semi-stable sheaves of small rank, preprint.

[6] Matsumura, H., On algebraic groups of birational transformations, Lincei Rend. Sc. fis. e nat., 34 (1963), 151-155.

[7] Morikawa, H., A note on holomorphic vector bundles over complex tori, Nagoya Math. J., 41 (1971), 101-106.

[8] Mukai, S., Duality between $D(X)$ and $D(\hat{X})$ with its application to the Picard sheaves, preprint.

[9] Mumford, D., Introduction to algebraic geometry, to appear.

[10] —- Abelian Varieties, Oxford Univ. Press 1970.

[11] Takemoto, F., Stable vector bundles on algebraic surfaces II, Nagoya Math. J., 52 (1973), 29-48.

[12] Ueno, K., Classification theory of algebraic varieties and compact complex spaces, Lecture notes in Math. 439, Springer (1975).

[13] Umemura, H., Some result in the theory of vector bundles, Nagoya Math. J., 52 (1973), 173-195.

[14] - Stable vector bundles with numerically trivial Chern classes over hyperelliptic surfaces, Nagoya Math. J., 59 (1975), 107-134.

[15] - On a certain type of vector bundles over an abelian variety, Nagoya Math. J., 64 (1976), 31-45.

[16] - On a property of symmetric products of a curve of genus 2, Proc. Int. Symp. Algebraic Geometry, Kyoto (1977), 709-721.

[17] Weil, A., Variétés abéliennes et courbes algebriques, Hermann, Paris 1948.

[18] — Z Zum Beweis des Torellishen Satzes, Nachr. Akad. Wiss. Göttingen 1957.

Nagoya University 